\title{
Review
}

\section{Ice breaking in GPCR structural biology}

\author{
Qiang ZHAO*, Bei-li WU \\ The Joint Laboratories of GPCR Research, Center of Structures and Functions of Drug Targets, Shanghai Institute of Materia Med- \\ ica, Chinese Academy of Sciences, Shanghai 201203, China
}

\begin{abstract}
G-protein-coupled receptors (GPCRs) are one of the most challenging targets in structural biology. To successfully solve a high-resolution GPCR structure, several experimental obstacles must be overcome, including expression, extraction, purification, and crystallization. As a result, there are only a handful of unique structures reported from this protein superfamily, which consists of over 800 members. In the past few years, however, there has been an increase in the amount of solved GPCR structures, and a few highimpact structures have been determined: the peptide receptor CXCR4, the agonist bound receptors, and the GPCR-G protein complex. The dramatic progress in GPCR structural studies is not due to the development of any single technique, but a combination of new techniques, new tools and new concepts. Here, we summarize the progress made for GPCR expression, purification, and crystallization, and we highlight the technical advances that will facilitate the future determination of GPCR structures.
\end{abstract}

Keywords: G-protein-coupled receptors (GPCRs); expression study; mutagenesis; surfactant; crystallization; bicelle; lipidic cubic phase

Acta Pharmacologica Sinica (2012) 33: 324-334; doi: 10.1038/aps.2011.187; published online 30 Jan 2012

\section{Introduction}

Membrane proteins comprise approximately $30 \%$ of organism proteomes; however, they comprise only $1.1 \%$ of the total protein data bank (PDB) entries (862 coordinate files in a total of 75694 depositions). Among which, the GPCRs are greatly under-represented in the PDB when compared to the other general protein families. These receptors are involved in every physiological activity, including sight, taste, and hormone regulation, etc. They function as signal transmitters that can sense a huge variety of signals and amplify them inside the cells, leading to different cell responses, and are involved in almost all human diseases. Among the approximately 400 non-olfactory receptors, 50-60 receptors are the targets of approximately $40 \%$ of the drugs on the market, while the other 350 are yet to be explored. As the largest protein family of drug targets in the human body, there are only 45 deposited structure files representing 7 unique receptors ${ }^{[1-7]}$. Moreover, this set is somewhat redundant, as the $\beta 1$ and $\beta 2$ adrenergic receptors are closely related. Given that over 800 receptors are found in the human genome and that a large variety of ligands specifically bind to this protein family, the mechanisms of ligand recognition and signal transduction are largely uncharacterized.

To successfully solve a GPCR structure, one needs to over-

\footnotetext{
* To whom correspondence should be addressed.

E-mail zhaoq@mail.shcnc.ac.cn

Received 2011-10-08 Accepted 2011-12-06
}

come multiple bottlenecks, including expression, extraction, and the formation of crystal contacts. In addition to these general difficulties that have been long known in crystallizing membrane proteins, the flexibility of the receptor poses a major obstacle ${ }^{[8,9]}$. It has been observed repeatedly for multiple receptors that the transmembrane helix bundle rearranges during activation. The distances between the helices differ by up to $10-14 \AA$ during this process ${ }^{[10-12]}$. It has also been reported that between the active and inactive states, these receptors have multiple intermediate states that are induced by different types of ligands, and each state might correlate to its own structural features. Because GPCRs are so flexible, they are generally in a mixture of different conformations, and the field tends to use a concept of "energy landscape" instead of "conformation" to describe the dynamics of GPCRs ${ }^{[13]}$. It is not uncommon to observe that exchanging the ligand or mutating one amino acid of the GPCR can dramatically reduce the diffraction resolution or even abolish crystallization altogether. This is probably one of the key reasons why it took researchers an additional 15 years after the first highresolution membrane protein structure was reported in $1985^{[1]}$ to obtain the first GPCR structure. Despite the current low success rates for achieving high-resolution structures, years of technology developments and studies of this protein family have shed light on this challenging project. Recent breakthroughs include the structures of novel GPCRs ${ }^{[7]}$, discovery of new activity states of GPCRs ${ }^{[10,14-16]}$ and a structure of GPCR in complex with other proteins ${ }^{[11]}$. This review will focus on new 
clues and the latest progress on the structure determination of GPCRs.

The experimental procedure for obtaining a membrane protein crystal structure typically involves three steps: the protein has to be functionally expressed, purified in detergent micelles and crystallized. We will summarize the recent technological developments accordingly.

\section{Protein expression}

With few exceptions, GPCRs are often found in very small amounts in native tissue, which makes it nearly impossible to purify the amounts of material necessary for crystallographic studies from natural sources. It is therefore necessary to set up a robust recombinant expression system. To achieve high yields of recombinant GPCR proteins, gaining a better understanding of the host organisms is an emerging strategy compared to standard techniques such as the screening of promoters, development of fusion adducts, and adjusting the culture process parameters. Conventionally, there are 4 types of expression host systems used in protein X-ray structural studies: E coli, yeast, insect cells and mammalian cells, and each system has its own limitations that restrict its use in GPCR overexpression studies.

\section{Mammalian cells and insect cells}

Among the four systems, the mammalian cell expression system is the closest to the natural environment of GPCRs, and thus serves as an expression host for many GPCRs. The yield of some receptors in this system is as high as $10 \mathrm{mg} / \mathrm{L}$ of media ${ }^{[17]}$; however, most of the receptors are designed for functional studies so far, and only a few of those were applied to overexpression. High cost, difficulty in scaling up and long experimental cycles are barriers that limit the use of mammalian cell expression systems.

On the contrary, insect cells, which shares similar disadvantages as mammalian systems, are a more successful host. Six out of seven unique receptors were expressed in either sf9 cells or high5 cells ${ }^{[18]}$. After careful optimization, the baculovirus expression system often has increased protein yields over that of mammalian cells such as HEK cells ${ }^{[19]}$.

\section{Yeast}

Yeast is an attractive expression system because it can be engineered as a microorganism while possessing eukaryotic machinery ${ }^{[20]}$. Yeast has a short generation time (approximately $2 \mathrm{~h}$ ), requires relatively simple media and is easy to work with. Years of characterization have also provided numerous plasmids and experience in scale-up fermentation. As a eukaryotic expression system, yeast can post-translationally modify the receptor; therefore, it was one of the earliest expression systems used for GPCR. However, the yeast system has its own drawbacks. The composition/ quantity of N-glycans and the membrane composition of yeast are different from those of mammalian systems. This may cause problems when a correct modification or lipid environment is necessary for the functional expression of some recep- tors, such as rhodopsin or opioid receptors ${ }^{[21,22]}$. In addition, yeast cells are surrounded by a cell wall that is notoriously hard to handle when trying to extract membrane and intracellular proteins.

Initial attempts were made in yeast systems, such as $S$ cerevisiae, $S$ pombe and $P$ pichia, to express the $\beta_{2}$ adrenergic receptor, the M1 muscarinic receptor and the opioid receptors ${ }^{[23-25]}$. Multiple factors including supplementation of a ligand, histidine and DMSO were evaluated and optimized ${ }^{[26,27]}$, and quite a few receptors were reported with expression levels of $>1 \mathrm{mg} / \mathrm{L}_{\text {of media }}{ }^{[28,29]}$, which is sufficient for structural studies. Recently, Iwata's group solved the crystal structure of the human Histamine $\mathrm{H} 1$ receptor, which was expressed in P pichia ${ }^{[7]}$. To avoid the complication of saccharide modification in the yeast system, the N-terminal portion comprising 19 amino acids which includes two glycosylation sites (Asn5 and Asn18) were truncated. The receptor sequence was also optimized according to the codon usage for P pichia to achieve a higher expression level ${ }^{[30]}$. The structure had very high similarity to the structure of $\beta_{2}$ adrenergic receptor and the D3 dopamine receptor, which were obtained using higher eukaryotic expression systems ${ }^{[7]}$. These results indicate that yeast has the ability to correctly fold mammalian GPCRs and has the potential for applications in protein crystallographic research.

Despite this tremendous success, we do not completely understand this expression system yet. Research from different groups raises our concern about the homogeneity of yeast-expressed material ${ }^{[8,31-33]}$. Their results indicate that the expressed product is a mixture of functional and nonfunctional receptors, because during optimization the expression of the functional GPCR will dramatically increase but the overall expression level is somewhat constant. In addition, the expression of GPCRs is case-sensitive. Although multiple receptors showed a relatively high expression of $1-10 \mathrm{mg} / \mathrm{L}$ of culture, the ratio of functional expression is extremely low. O'MALLEY et al investigated 12 GPCRs expressed in $S$ cerevisiae, and only the $\mathrm{A}_{2 \mathrm{~A}}$ adenosine receptor retained its ligand binding activity. Other receptors, despite being processed and expressed in the same conditions, failed to localize in the plasma membrane and had no detectable ligand binding ${ }^{[31]}$. A more detailed study using $\mathrm{N}$-terminal sequencing and $\mathrm{N}$-glycosylation detection demonstrated that most of these nonfunctional receptors were not properly processed. In addition to $S$ cerevisiae, $P$ pichia had similar problems in processing signal sequences ${ }^{[34]}$, indicating that there are still some fundamental differences within the higher eukaryotic systems. Currently, little is known regarding the factors that govern the folding and cellular trafficking of heterologously expressed GPCRs in yeast, and more efforts are needed in the future to make this system more robust and desirable.

\section{E coli}

Although it is known that eukaryotic membrane proteins, especially GPCRs, are impossible to express efficiently in prokaryotic systems, the low-cost and easy-to-handle nature of $E$ coli encouraged the exploration of this system for the 
expression of GPCRs. Initially, researchers managed to make some progress by successfully obtaining GPCRs such as the $\beta_{2}$ adrenergic receptor and the 5-hydroxtryptamine receptor (5HT1a) with functional ligand-binding activity ${ }^{[35]}$. The key was to use a maltose-binding protein (MBP) as an N-terminal fusion tag, which helped the receptor to be expressed and folded in the periplasm. Grisshammer et al also found that addition of thioredoxin A to the C-terminus of GPCR further increased their stability and yield ${ }^{[36]}$.

However, even if all of the expression parameters are optimized, the GPCRs expressed in E coli may not be sufficient for direct use in crystallographic studies yet. To date, most crystallization studies are focused on indirect uses, such as expression or stability screening, to facilitate higher throughput in a short period of time. The best example of this was provided by the Tate group in solving the crystal structures of the $\beta 1$ adrenergic receptor and the $\mathrm{A}_{2 \mathrm{~A}}$ adenosine receptor in the active state ${ }^{[16,37]}$. All of the mutants were expressed in $E$ coli using a procedure that is similar to Grisshammer's, and the construct optimization was performed accordingly, before the final transfer to insect cells for large-scale preparations. The work of PLÜCKTHUN et al pushes these approaches to a new level. They developed a fluorescence-activated cell sorting (FACS) method that could enhance both the expression level and stability of GPCRs while retaining function and tailoring ligand selectivity ${ }^{[38]}$. Using this approach, the expression levels of multiple GPCRs were increased several folds, and a receptor analog that more prone to bind agonists $v$ s antagonists was obtained, similar to results from the Tate group when they solved the active state $\mathrm{A}_{2 \mathrm{~A}}$ adenosine receptor structure ${ }^{[39]}$. Moreover, data from both the Tate and Plückthun groups indicate that all the results from prokaryotic systems can be successfully transferred to eukaryotic expression systems. This is truly exciting because one can take advantage of the reduced cost, the lower demand for experience and the dramatically shorter experimental cycles of prokaryotic systems. However, although this approach is very promising now, the application of prokaryotic expression systems for structural studies requires further development.

Because certain GPCRs are currently produced at sufficient levels for X-ray crystallographic trials, it is speculated that heterologous expression of GPCRs may no longer be a bottleneck in obtaining crystal structures. However, there remain many unknown factors that are important for optimizing GPCR expression. It is not surprising to see $>100$ fold differences in yield among various GPCRs within each of the expression systems. While milligram quantities of certain GPCRs are attainable, the majority of GPCRs are still either produced at very low levels or not at all. Developing reliable expression techniques for GPCRs is still a major priority for the structural characterization of GPCRs.

\section{It's all about stability}

Once acceptable expression is achieved, the issue of extracting the receptor out of the lipid bilayer starts to limit the downstream processes toward crystallization. Most of the GPCRs are intrinsically unstable and quickly lose their native fold when solubilized ${ }^{[9]}$. It is generally known that protein stability is correlated to the chances of protein crystal formation, and this point has been repeatedly demonstrated by the Tate and Stevens groups. Multiple methods have been carried out to engineer the receptor for higher stability.

\section{T4 lysozyme fusion}

The most successful attempt at engineering a GPCR to solve its structure is the generation of a T4 lysozyme fused protein. This was first developed during solving the high-resolution structure of the $\beta_{2}$ adrenergic receptor ${ }^{[2,40]}$. It has long been known that the third intracellular loop is very flexible, and is believed preventing the formation of crystals. Replacing of this loops with the T4 lysozyme moiety will not only provide an additional surface for crystal contact, but also restrain the movement of the GPCR helix bundle and thus provides higher thermal stability ${ }^{[40]}$. Research also revealed that this replacement does not alter either the GPCR's ligand binding or signal transduction ability. Subsequent research showed that this T4 lysozyme (T4L) fusion method is applicable to different GPCRs, even though the junction sites are slightly different and must be carefully screened ${ }^{[5,6]}$.

Different methods are used to determine the best T4L fusion. Initially, the insertion site was decided by receptor surface expression screening. Together with determination of more GPCR structures, a more systematic screening method combining ligand-binding affinity, protein homogeneity and stability was developed $^{[5,6,41]}$. Guided by this method, several structures were successfully solved including the chemokine receptor CXCR4 and the dopamine receptor D3.

\section{Antibodies/nanobodies}

The application of antibodies is a traditional method for dealing with flexible/instable proteins. So far, two GPCR structures have been solved in the presence of antibodies that recognizing the receptor ${ }^{[10,42]}$, both of which are $\beta_{2}$ adrenergic receptor structures. Similar with the use of the T4 lysozyme fusion, these antibodies target the cytoplasmic side of the receptor, especially the unstructured intracellular loop 3 (ICL3) region. In the $\beta_{2} \mathrm{AR}-\mathrm{Fab5}$ complex structure, the Fab5 recognizes both the junction of the helix V to ICL3 (I233-V242) and the junction of ICL3 to helix VI (L266 and K270) ${ }^{[42]}$, which will restrain the movement of these two helices. Furthermore, in the $\beta_{2} \mathrm{AR}-\mathrm{Nb80}$ complex, the nanobody $\mathrm{Nb80}$ also specifically recognizes both helix $\mathrm{V}$ and helix VI in the cytoplasmic end. In addition, its CDR3 loop inserted into the hydrophobic pocket between helices V/VI and helices III/VII ${ }^{[10]}$. These antibodies do help with the formation of crystal contacts, but their role in restricting the receptor dynamics is equally important. It looks like the helices V and VI are the key element to be stabilized before the receptor is actually crystalized.

This method do improve our chances of getting crystals, however, the application of antibodies for GPCRs is not straightforward. One major obstacle is the existence of very high concentration of detergents. These receptors generally 
require higher protein concentrations for crystallization (30-50 $\mathrm{mg} / \mathrm{mL}$ or higher), which typically requires at least a 100 -fold concentration. As a result, the detergent concentration in the final crystallization samples is strikingly high (approximately $2 \%$ in the case of DDM), and some of these antibodies will dissociate from the receptor molecules under such conditions. The fact that only the $\beta_{2}$ adrenergic receptor has been crystallized with the help of antibodies indicates that this method is equally challenging, and extensive work is needed before practical use.

\section{Mutations}

Besides the lysozyme fusion and antibodies, additional mutations are generally needed to facilitate crystallization. The most common is a E3.41W mutation, which was first introduced in the $\beta_{2}$ adrenergic receptor (the amino acid is represented using the Ballesteros-Weinstein numbering-system ${ }^{[43]}$ ). In the sequence alignment of $\beta_{2}$ adrenergic receptor with other GPCRs, there is an hydrophilic glutamate residue in the middle of helix III that is buried in the hydrophobic helix bundle $\mathrm{e}^{[44]}$ (Figure 1B), which might reduce the stability to the whole receptor. Mutation of this residue to an aromatic residue dramatically increases the yield of the purified mate- rial and greatly improves the receptor's melting temperature. Additional research showed that similar to the T4 lysozyme fusion, this mutation could also be applied to other receptors, even if their corresponding positions are already hydrophobic residues $^{[5,6]}$ (Figure 1).

In some cases, although the mutations themselves make the receptor stable enough to be crystalized, it remains difficult to explain the rationale for these mutants. Tate and Schertler have mapped almost every residue in the receptor and measured the thermal stability of the corresponding mutants. The most promising mutations were then combined and further screened for the highest stability to tolerate the harsh environment during extraction, purification and crystallization ${ }^{[38,45]}$. Guided by this method, they solved the crystal structures of the $\beta 1$ adrenergic receptor with different ligands and the $A_{2 A}$ adenosine receptor structure in an active state ${ }^{[4,16]}$. Similarly, PLÜCKTHUN et al used error-prone PCR (epPCR) to construct a library of mutations of target gene which were expressed and sorted by flow cytometry to screen for higher expression or stability. The sorted cells were kept and then epPCR and pooled for 4 rounds before they were analyzed for sequence diversity. The most consistent mutations were selected and successfully applied to higher expression systems such as
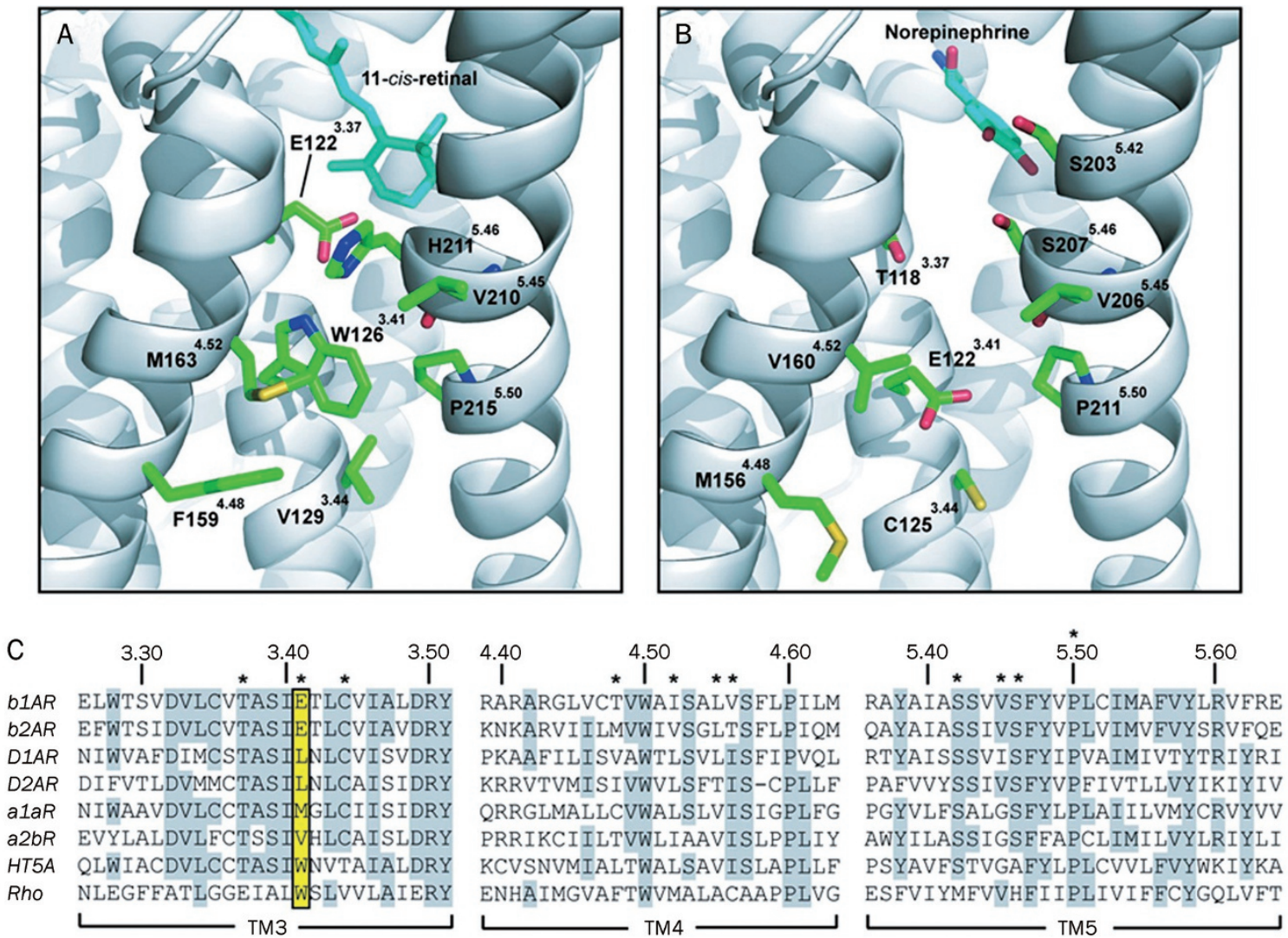

Figure 1. Structural models of the TM4-3-5 interface, indicating the most frequent 3.41 (Ballesteros and Weinstein System) mutation in GPCR structures. (A, B) Rhodopsin inactive state structure (PDB ID code 1U19) and $\beta_{2} A R$ structure showing residues proximal to 3.41 . TM helices are colored grey and side-chains carbon atoms are colored green. (C) Clustal W sequence alignment illustrating the residue conservation in TM3, TM4, and TM5 for the BARs, rhodopsin and several members of the biogenic amine family. Identical residues are highlighted in grey. Key residues mentioned in the text are marked with asterisks. Position 3.41 is highlighted in yellow ${ }^{[44]}$. 
insect cells, and by this, higher expression and stability were then achieved ${ }^{[38,39]}$.

These three groups tested stabilization mutant using 5 GPCRs independently and found some very interesting consistencies ${ }^{[37-39,45,46]}$. Each of the five receptor genes contained $5.6 \pm 1.1$ mutants to maximize the protein stability compared to the wild type, with a total 28 mutants. The majority of these mutations are located in the helix bundle ( 26 out of 28 mutations), and they are not spread evenly on all the seven transmembrane helices (Figure 2). Nearly half of the mutations are distributed from helix V through helix VII, where there was significant movement upon activation. Surprisingly, helix II is also one frequently mutated region. More than $30 \%$ of the stabilizing mutations occurred on this helix (9 out of 28 mutations) for no obvious reason. The other helices, especially helix IV, are barely touched which is probably due to it is nowhere near helix V, VI, or VII in the spatial helix bundle. Although only a few mutations appeared on identical positions, the majority of the mutations lie only in several specific pockets. These pockets are located in the intracellular end of the receptor, and only a few mutations are located in the extracellular half that facilitates the binding of certain ligands. This is not completely unexpected, as research showed that the intracellular portion displayed more structural dynamics in different active state. In contrast, the extracellular half, despite having significant diversity within the gene superfamily, has only very minor changes when different ligands are bound.

GPCRs have enormous conformational diversity, which is believed to be one of the key obstacles to crystallization. To date, all the receptor analogs achieved from mutation screening had a modified binding preference depending on the type of ligands used ${ }^{[4,16]}$. For example, in the structure of the $\mathrm{A}_{2 \mathrm{~A}}$ adenosine receptor bound to agonists adenosine and NECA, four mutations in the receptor greatly reduced the affinity with the antagonists, while the agonist binding was unaltered ${ }^{[16]}$. The shift in binding affinity indicates that the receptor could only adopt restrained conformations, thus its stability and homogeneity were improved, which would further allow crystallization. Because most of these conformational restraining mutations are located in the intracellular portion of the GPCR where they are more conserved in both structure and sequence than the other regions, further studies could potentially reveal a general rule that could apply to the other GPCRs and guide our future studies.

\section{Surfactants}

Traditionally, short-chain detergents form smaller micelles around membrane proteins than the long alkyl ones, which would leave larger surface areas exposed to form crystal contacts. However, these short-chain detergents are also far more denaturing than the long-chain detergents that are normally used to purify GPCRs in a functional form. It is very challenging to choose a detergent that can balance the hydrophobicity and hydrophilicity for each target protein, and as a result, the crystals are often of low quality and difficult to improve. Based on the understanding achieved from previous X-ray crystallographic work, several new amphiphiles and detergents have been developed by modifications of known surfac-

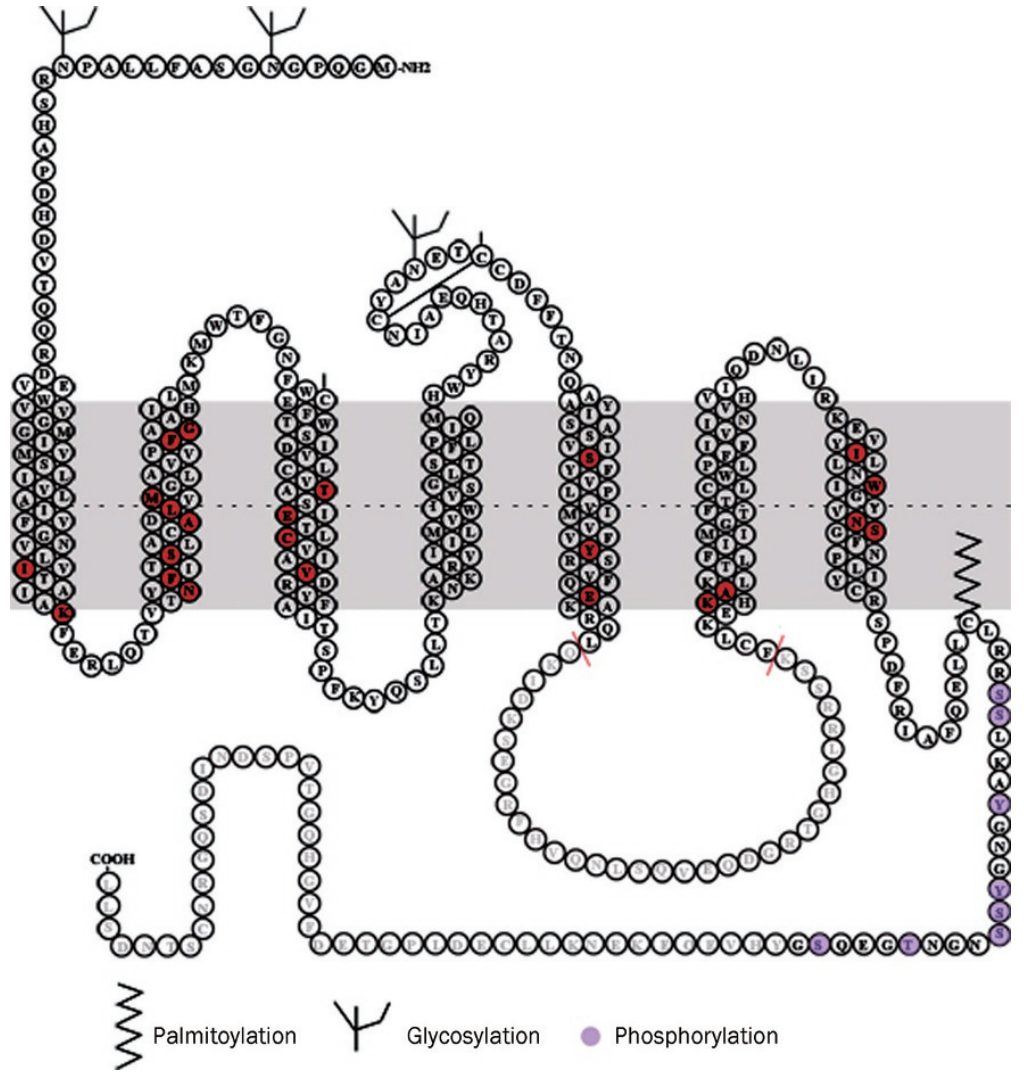

Figure 2. A snake $\beta_{2} A R$ plot to brief summary of the mutagenesis studies aiming higher GPCR stability ${ }^{[37,38,44-46,91]}$. The mutations on different receptors are applied to $\beta_{2} A R$ receptor based on the Ballesteros and Weinstein Number System ${ }^{[43]}$ and labeled in red on the Figure. The post translational modifications such as glycosylation and phosphorylation are labeled as indicated. 
tants or de novo design. Some of these novel amphiphiles (eg, nanodiscs and amphipols) have found broad application in membrane protein biochemistry and are used for solubilization and stabilization of GPCRs for functional studies.

One example of these modified detergents is branch-chained detergent which contains a short, branched alkyl chain at the interface between the polar head and the apolar tail. This type of detergent mimics a lipid molecule with a second aliphatic chain, reducing the water penetration. Hong et al showed that even one carbon branch could have dramatic effects and these detergents could be successfully applied to solubilization, stabilization and crystallization of membrane proteins ${ }^{[4]}$. Another example of such detergents is maltose-neopentyl glycol (MNG) amphiphiles that are built around a central quaternary carbon atom ${ }^{[48]}$ (Figure $3 \mathrm{~A}$ and $3 \mathrm{~B}$ ). This carbon atom enables the incorporation of two hydrophilic and two hydrophobic subunits and restrains their conformational flexibility. Some of these MNG detergents have been tested on both GPCRs and other membrane proteins, and proved to enhance structural stability and the chances of successful crystallization. The authors also claimed that these detergents improved the crystal size and quality of membrane proteins with less stability, such as $\beta_{2} \mathrm{AR}-\mathrm{T} 4 \mathrm{~L}$ bound to agonists. In short, these modified detergents displayed distinct properties from their conventional analogs and have promising use in the mainte- nance of native GPCR folding and function ${ }^{[48]}$.

The de novo synthesis of detergents includes protein-based nanodiscs ${ }^{[49,50]}$, amphiphilic polymers (amphipols) ${ }^{[51-53]}$, peptide-based amphiphiles ${ }^{[54-56]}$, fluorinated detergents ${ }^{[53,57]}$ and tripod detergents ${ }^{[58,59]}$. One good example is the socalled "facial amphiphile", which is a new detergent concept created by several groups. These detergents make the protein-detergent complexes (PDC) as small as possible by creating a flat and rigid hydrophobic surface. McGregor et al have reported that lipopeptides (LPDs) are self-assembled into a cylindrical micelle with a width similar to that of a lipid bilayer, and further form a rigid sheath around the protein surface ${ }^{[55]}$. Zhang et al have developed cholate-based amphiphiles that project hydrophilic maltose units from one side of the steroidal skeleton, which is then further developed by the design of "tandem facial amphiphiles (TFAs)" ${ }^{\prime[60,61]}$. The TFAs contain a pair of maltose-functionalized deoxycholate units, making it long enough to match the bilayer width. These facial amphiphiles are very successful in reducing the micelle size. Both Zhang et al and Chae et al showed that the facial amphiphiles are only approximately $1 / 6$ of the micelle size compared to the traditional detergents such as DDM, with an aggregation number of approximately 37 for FAs and only approximately 6 for the TFAs per PDC ${ }^{[6,61]}$. Although there is yet to be a direct application of these facial amphiphiles in

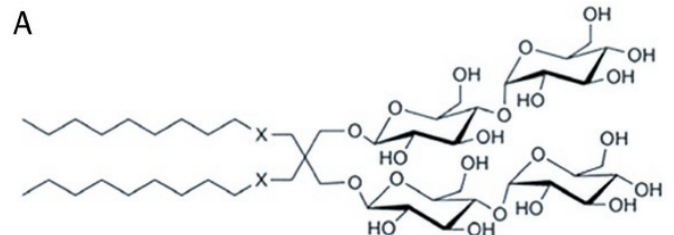

C

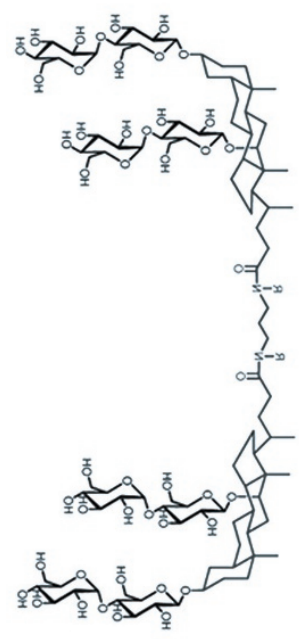

D
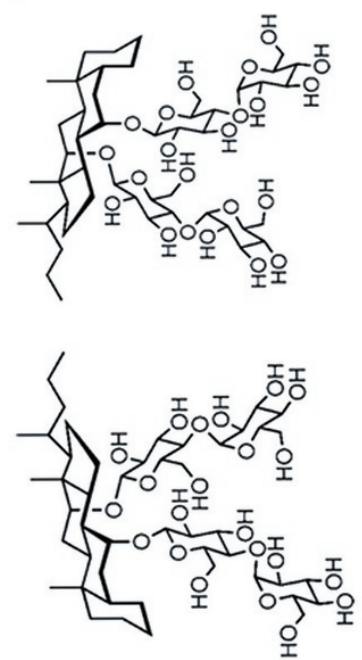

B

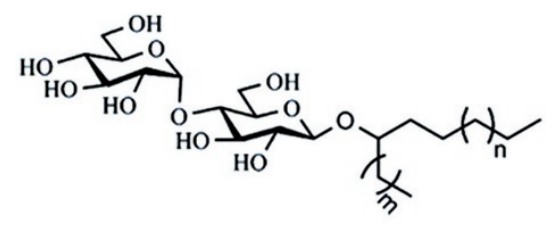

$E$
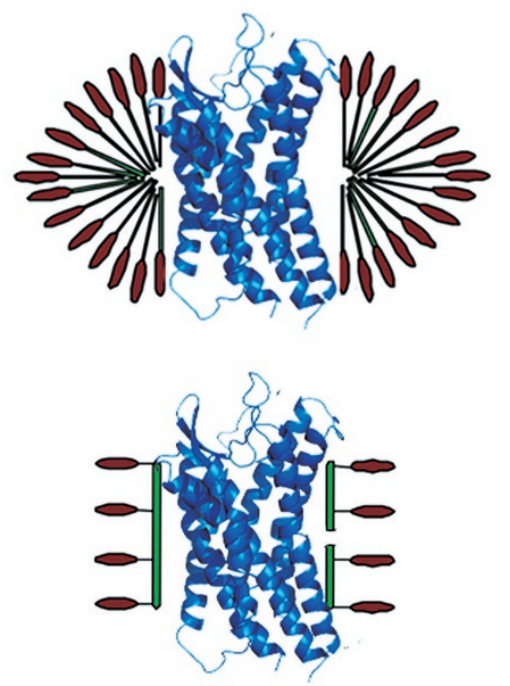

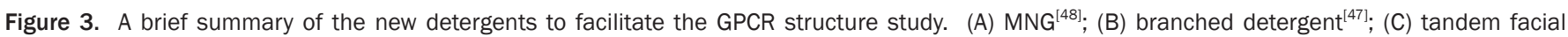

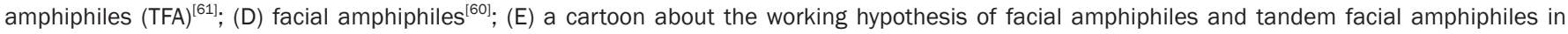
compare with conventional detergents ${ }^{[60]}$. 
crystallization trials, their biophysical properties exhibit significant potential in the structural study of this challenging protein family (Figure 3C, 3D, and 3E).

Recently, systematic screening of the branched detergents, MNGs and the facial amphiphiles for protein thermal stability was carried out using multiple GPCRs. More than half of these new detergents provided equal or higher thermal stability than the best conventional detergents (unpublished data). However, different GPCRs still have different preferences for different surfactants, and it seems very unlikely that one single amphiphile is ideal for all of the receptors due to their sequence variety. A deeper understanding of the interaction between membrane proteins and detergents is still required for further development of detergents.

\section{Crystallization and data collection}

To solve the structures of GPCRs, high-quality crystals must be obtained, which represents a major challenge. Several techniques are employed to crystallize GPCRs, including the traditional direct use of solubilized protein-surfactant complexes, or the so-called in surfo method ${ }^{[4,16]}$, the bicelle method ${ }^{[10,15]}$, and the lipidic cubic phase, or in meso method.

\section{In surfo method}

Although the in surfo method remains one of the most efficient in membrane protein crystallography, it exhibits dramatic limitations in GPCR structural studies. Research has shown that GPCRs tend to reserve more conformations in detergent micelles than in lipid bilayers, and as a direct result, very few GPCRs could crystallize directly in detergent micelles. Most of the current GPCR structures obtained using the in surfo method are modified by mutation to reduce their chances of adopting other conformations ${ }^{[4,16]}$ as mentioned above.

\section{Bicelle method}

The other methods, such as the newly developed bicelles, nanodiscs or lipidic cubic phase (LCP), seek to trap each membrane protein molecule within a lipid bilayer before crystallization and take advantage of a native-like environment to preserve their integrity ${ }^{[62]}$. Compared to the in surfo method, the bicelle method uses a lipid bilayer of finite size and maintains the ability to diffuse in three dimensions in the process of forming a three-dimensional lattice (Figure 4A). Bicelles are typically made of two lipids, one of which forms a lipid bilayer, while the other forms an amphipathic, micelle-like cover for the bilayer and shields it from the solvent ${ }^{[63]}$. The important interactions between lipids and proteins have been preserved within bicelles, as clear density for a CHAPSO molecule inserted between protein subunits is observed in the structure of bacteriorhodopsin. The bicelles have displayed broad utilities in every aspect of GPCR study ${ }^{[64]}$. The ability to grow crystals at room temperature significantly expands the applicability of bicelle crystallization ${ }^{[42,65]}$. Nanodiscs, on the other hand, use an amphipathic protein coat to encapsulate the bilayer segment instead of detergent, which makes nanodiscs more stable than bicelles at low concentrations ${ }^{[49,66]}$.
The nanodisc is constrained by two molecules of a membrane scaffold protein that wraps around the edges of the discoidal structure in a belt-like arrangement. These constructs maintain a very well-defined size depending on the type of protein coat $^{[67]}$. Because the smallest nanodiscs are approximately 10 $\mathrm{nm}$, their application in crystallography is still quite limited, and more work needs to be performed to remove the 3-D packing restraints before successful crystallization. To date, these bilayer-based, diffusible structures have been most useful for NMR methods or for assessing the ligand binding of GPCRs $^{[67-69]}$.

\section{In meso method}

Crystallization in lipidic phases has only recently been developed but has already become an essential tool in the arsenal of membrane protein crystallization, especially for GPCRs ${ }^{[70,71]}$. The cubic phase is a bicontinuous lipidic meso phase formed spontaneously by mixing monoacylglycerols (MAGs) and water at a given ratio ${ }^{[72]}$ (Figure 4B). It consists of a curved bicontinuous lipid bilayer in three dimensions, separating two congruent networks of water channels. When protein samples are used as an aqueous solution, the high concentration of lipid molecules will replace the detergent molecules around the protein, and thus, the receptor is reconstituted to the lipid bilayer. When a certain precipitant is added, the receptor will start to nucleate, and a temporary phase transition will be formed, ensuring crystal growth (Figure 4C).

Monoolein is a default lipid for crystallogenesis studies. However, some other lipids such as monomyristolein (C14:1c9) and monopentadecenoin (C15:1c10) could also be used for crystallization studies. The different host MAGs, or MAGs with different additives (usually cholesterol or phospholipids), come with varying properties including hydrophobic thickness, intrinsic curvature, and lateral pressure profile $^{[73,74]}$. These lipids have shown a profound effect upon nucleation, crystal shape and diffraction in different targets ${ }^{[73]}$. This method originated from Ehud Landau and Jürg Rosenbusch in 1996 and was soon followed by application to bacteriorhodopsin ${ }^{[75,76]}$, the $\beta 2$ adrenergic receptor ${ }^{[2]}$, the chemokine receptor $\mathrm{CXCR} 4^{[5]}$, and the dopamine receptor $\mathrm{D} 3^{[6]}$.

It should be safe to assume that during the conditions of crystallogenesis, receptor molecules have to retain a certain level of freedom to diffuse so that nucleation and crystal growth can occur. As mentioned above, we learned that the lipidic cubic phase is composed of highly curved lipid bilayers and is connected by a water channel of a certain size ${ }^{[77]}$. As proteins diffuse through the cubic phase, they encounter horse saddles of high Gaussian curvature ${ }^{[78]}$. In some cases, one could find their protein stuck inside the lipid bilayers, either due to the energy barrier of crossing the horse saddle and the monkey saddle, or due to the limited size of the water channel. Several groups have tried to build a computational model, but they had little success ${ }^{[78,79]}$. The reason for failure might be that there are several components in the crystallization trials, quite a few of which, such as salt, PEGs and trace amounts of detergent, could alter the dimensions or even the phase behav- 
A

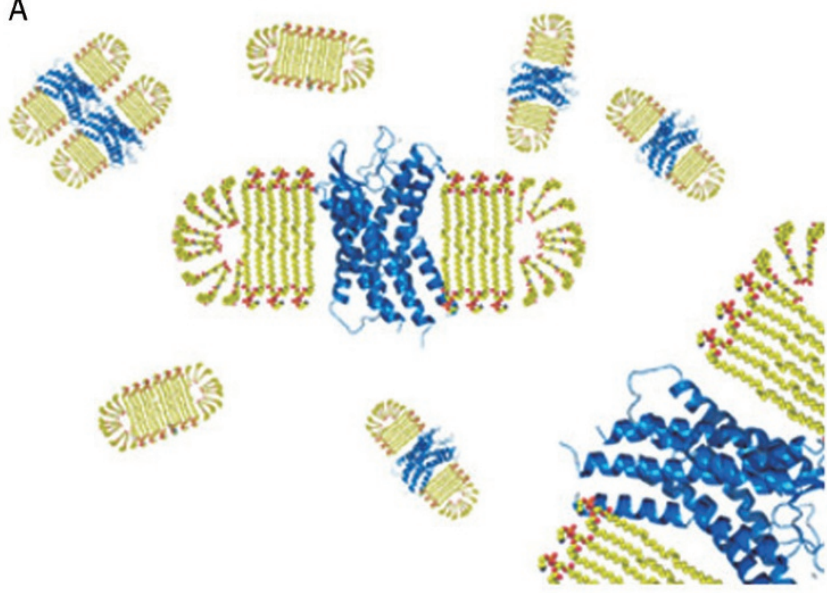

C

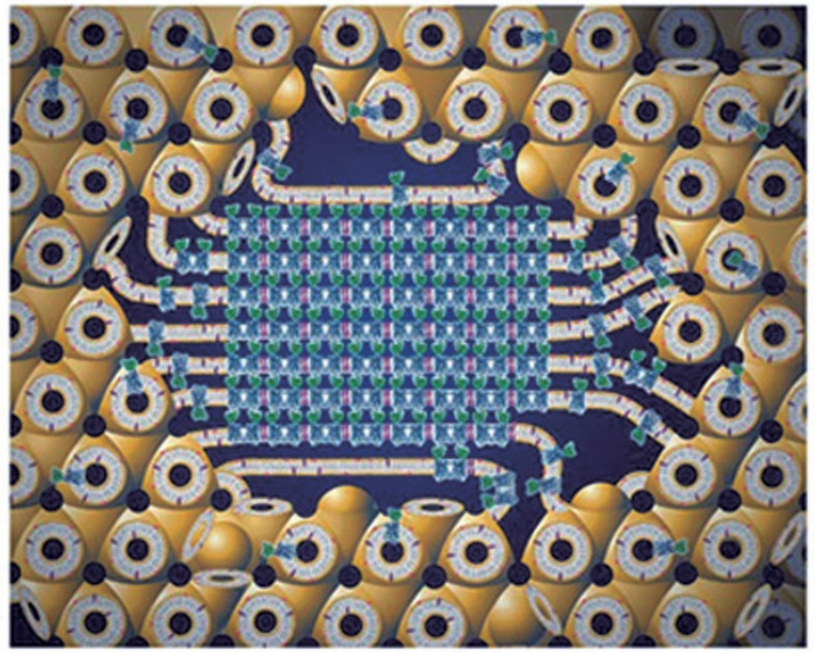

B

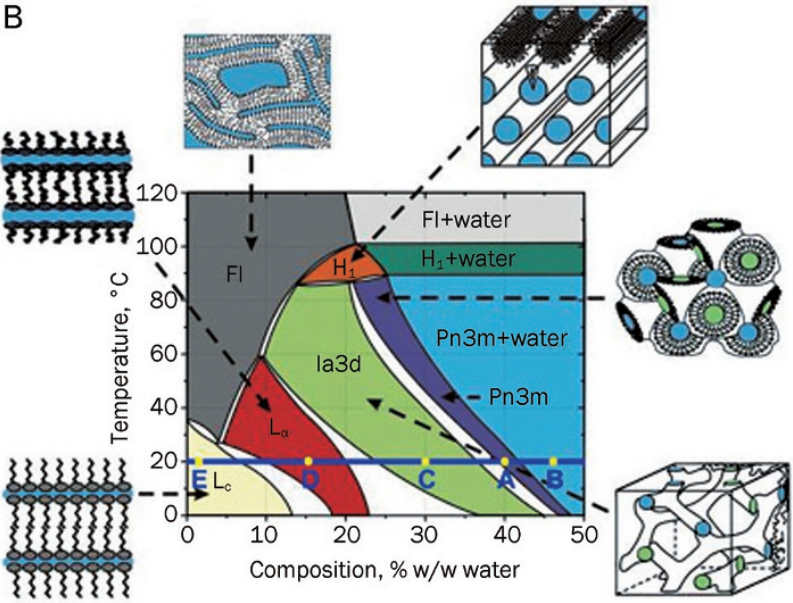

D

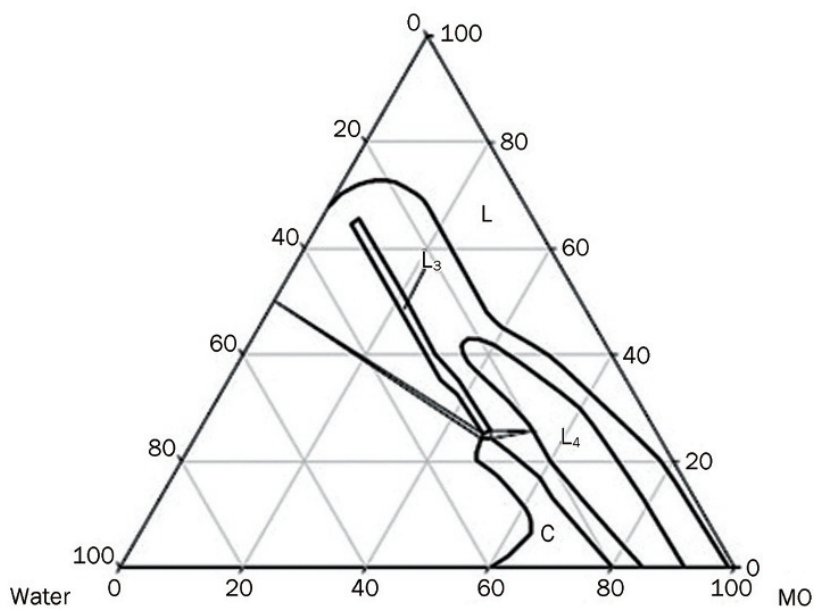

Figure 4. Phase diagram of lipidic cubic phase use Monoolein. A) A representative figure of GPCR in bicelle. The receptor is surrounded by a DMPC lipid bilayer, which is further covered by detergents. The receptor-lipid-detergent is afloat in the solution. D) the phase-temp diagram of Monoolein and water system ${ }^{[72]}$, the phase will transit as lipid:water ratio or temperature change; C) crystallization of GPCRs in cubic phase, the receptor is in cartoon, the bicontinuous lipid bilayer is drawn in yellow and white and the dark blue represents the water channel and aqueous solution; D) Phase behavior of lipid-water-PEG400 system, representing the influences of additives and lipids on the phase ${ }^{[81]}$.

ior of the whole system ${ }^{[80,81]}$ (Figure 4D).

Unlike the theoretical analysis, progress was made by experimental studies of the lipidic cubic phase guiding the crystallization studies. One powerful approach, fluorescence recovery after photobleaching (FRAP), seeks to assess the ability that GPCRs have while in the lipidic bilayer-based meso phase and identify conditions that favor diffusion in two dimensions as freely as possible to find other protein partners with which to build the two-dimensional array ${ }^{[82]}$. A major advance is the application of fluorescence to assay the diffusion rates, seeking conditions that maximize the diffusibility. Based on the FRAP method, high throughput FRAP (HT-FRAP), which combines traditional FRAP with an automation technique, was further developed and showed a dramatic advance in reducing the work load ${ }^{[83]}$. Instead of measuring the full recovery curve, the precise motion control allows it to scan a 96-well plate and compare exact spots at different time points, which increases the measurement efficiency by approximately 50 fold. This is a key element for the application of this technique to practical research. Using this method, one can now pre-screen host lipids, precipitant conditions, and identities of ligands with microgram quantities of material to magnify the chances of crystallogenesis and rule out conditions that are not conducive to diffusion, nucleation, and crystal growth ${ }^{[83]}$. LCP-Tm, another LCP tool to measure the stability of the receptor in the host's lipids without the use of labels, will also facilitate the screening of better constructs and ligands ${ }^{[84]}$. Assisted by different LCP tools, the variables of the multi-dimensional crystallization space are notably reduced, and thus, the bottleneck in obtaining initial crystal leads is substantially overcome.

Of course, like any other crystallization technique, the in meso method has its own drawbacks: it is relatively hard to handle, incompatible with some of the precipitants, rather small that are general invisible under cryo conditions. To 
make things worse, to generate crystals of sufficient quality so that their structure may be obtained, the lipidic cubic phase generally requires a higher receptor concentration $(>50$ $\mathrm{mg} / \mathrm{mL})^{[2,5,6,14]}$, which challenges the already troubled expression and purification protocols of GPCRs. Most of the above obstacles are diminished by the development of robotic systems and microfocus beams. Robotic crystallization technologies, which can dispense subnanoliter-scale drops in 96-well plates within minutes, have expended the use of the lipidic cubic phase by substantially increasing the number of crystallization conditions that can be explored with limited amounts of sample $\mathrm{e}^{[72,85]}$. Another recent innovation is the microfocus beamline at synchrotron ${ }^{[86]}$, which makes data collection from the undersized GPCR crystals possible. Smaller X-ray beams, reduction of the diffraction background, and an increase in the beam intensity remarkably improve the resolution and the data statistics compared to the general synchrotron beams, and allow useful diffraction data to be extracted from smaller crys$\operatorname{tals}^{[2,86]}$. Although tighter focus comes at the cost of greater radiation damage to the crystal, it can be overcome by merging data from several or even tens of crystals ${ }^{[2,6]}$ (hundreds in some cases, data unpublished) and newer detectors ${ }^{[87]}$. Some other techniques, although not as essential as microfocus beams or crystallization robots, significantly improve research efficiency. Sample-exchange robots that allow crystals to be replaced without entering the experimental hutch are showing more and more impact on X-ray diffraction data collection. Rapid crystal alignment and raster tools will further accelerate the systematic screening of invisible crystals in the cryo loops, and more crystals could be tested in a shorter amount of time ${ }^{[88-90]}$. With the combination of the benefits from all the above techniques, the lipidic cubic phase is becoming the most popular method in determining the structures of GPCRs.

\section{Future prospects}

Structure-based drug design targeting of G-protein coupled receptors has long been limited by the availability of highresolution receptor structures. However, this limitation is decreasing, as multiple GPCR structures have been determined. The year 2011 could be considered a new landmark for understanding this largest drug target family. In this year, not only have new GPCR structures been obtained, but also structures of activated receptors and even the magnificent structure of the GPCR-G protein complex. These structures, and the purified membrane proteins that are produced before the structures are resolved, will enable us to develop a rational approach to the treatment of cancer, autoimmune diseases, and infectious diseases that endanger human health. This new progress also indicates that we are starting to understand how GPCRs function in response to the binding of a natural ligand or a drug. This is also a direct result of the unremitting development of techniques during the last few years. The introduction of effective new tools for the membrane structural biologist reflects the ingenuity of the current researchers and lays the groundwork for applications to numerous diseases. It is safe to expect more structures in the near future as newer techniques, tools, chemicals and protocols will be applied to research that will further shorten the timeline and reduce the resources needed for solving a GPCR structure.

\section{References}

1 Palczewski K, Kumasaka T, Hori T, Behnke CA, Motoshima H, Fox BA, et al. Crystal structure of rhodopsin: A G protein-coupled receptor. Science 2000; 289: 739-45.

2 Cherezov V, Rosenbaum DM, Hanson MA, Rasmussen SG, Thian FS, Kobilka TS, et al. High-resolution crystal structure of an engineered human beta2-adrenergic G protein-coupled receptor. Science 2007; 318: 1258-65.

3 Jaakola VP, Griffith MT, Hanson MA, Cherezov V, Chien EY, Lane JR, et al. The 2.6 angstrom crystal structure of a human $A_{2 A}$ adenosine receptor bound to an antagonist. Science 2008; 322: 1211-7.

4 Warne T, Serrano-Vega MJ, Baker JG, Moukhametzianov R, Edwards PC, Henderson R, et al. Structure of a beta1-adrenergic G-proteincoupled receptor. Nature 2008; 454: 486-91.

5 Wu B, Chien EY, Mol CD, Fenalti G, Liu W, Katritch V, et al. Structures of the CXCR4 chemokine GPCR with small-molecule and cyclic peptide antagonists. Science 2010; 330: 1066-71.

6 Chien EY, Liu W, Zhao Q, Katritch V, Han GW, Hanson MA, et al. Structure of the human dopamine D3 receptor in complex with a D2/ D3 selective antagonist. Science 2010; 330: 1091-5.

7 Shimamura T, Shiroishi M, Weyand S, Tsujimoto H, Winter G, Katritch $\mathrm{V}$, et al. Structure of the human histamine $\mathrm{H} 1$ receptor complex with doxepin. Nature 2011; 475: 65-70.

8 Lundstrom K. Structural genomics of GPCRs. Trends Biotechnol 2005; 23: 103-8.

9 Bockenhauer S, Furstenberg A, Yao XJ, Kobilka B, Moerner WE. Conformational dynamics of single $G$ protein-coupled receptors in solution. J Phys Chem B 2011; 115: 13328-38.

10 Rasmussen SG, Choi HJ, Fung JJ, Pardon E, Casarosa P, Chae PS, et al. Structure of a nanobody-stabilized active state of the beta adrenoceptor. Nature 2011; 469: 175-80.

11 Rasmussen SG, DeVree BT, Zou Y, Kruse AC, Chung KY, Kobilka TS, et al. Crystal structure of the beta ${ }_{2}$ adrenergic receptor-Gs protein complex. Nature 2011; 477: 549-55.

12 Park JH, Scheerer P, Hofmann KP, Choe HW, Ernst OP. Crystal structure of the ligand-free G-protein-coupled receptor opsin. Nature 2008; 454: 183-7.

13 Deupi X, Kobilka BK. Energy landscapes as a tool to integrate GPCR structure, dynamics, and function. Physiology (Bethesda) 2010; 25: 293-303.

14 Xu F, Wu H, Katritch V, Han GW, Jacobson KA, Gao ZG, et al. Structure of an agonist-bound human $A_{2 A}$ adenosine receptor. Science 2011; 332: 322-7.

15 Rosenbaum DM, Zhang C, Lyons JA, Holl R, Aragao D, Arlow DH, et al. Structure and function of an irreversible agonist-beta(2) adrenoceptor complex. Nature 2011; 469: 236-40.

16 Dore AS, Robertson N, Errey JC, Ng I, Hollenstein K, Tehan B, et al. Structure of the adenosine $A_{2 A}$ receptor in complex with ZM241385 and the xanthines XAC and caffeine. Structure 2011; 19: 1283-93.

17 Reeves PJ, Kim JM, Khorana HG. Structure and function in rhodopsin: a tetracycline-inducible system in stable mammalian cell lines for high-level expression of opsin mutants. Proc Natl Acad Sci U S A 2002; 99: 13413-8.

18 Kempf J, Snook LA, Vonesch JL, Dahms TE, Pattus F, Massotte D. Expression of the human mu opioid receptor in a stable Sf9 cell line. J Biotechnol 2002; 95: 181-7. 
19 Hanson MA, Brooun A, Baker KA, Jaakola VP, Roth C, Chien EY, et al. Profiling of membrane protein variants in a baculovirus system by coupling cell-surface detection with small-scale parallel expression. Protein Expr Purif 2007; 56: 85-92.

20 Reilander H, Weiss HM. Production of G-protein-coupled receptors in yeast. Curr Opin Biotechnol 1998; 9: 510-7.

21 Kaushal S, Ridge KD, Khorana HG. Structure and function in rhodopsin: the role of asparagine-linked glycosylation. Proc Natl Acad Sci U S A 1994; 91: 4024-8.

22 Lagane B, Gaibelet G, Meilhoc E, Masson JM, Cezanne L, Lopez A. Role of sterols in modulating the human mu-opioid receptor function in Saccharomyces cerevisiae. J Biol Chem 2000; 275: 33197-200.

23 King K, Dohlman HG, Thorner J, Caron MG, Lefkowitz RJ. Control of yeast mating signal transduction by a mammalian beta 2-adrenergic receptor and Gs alpha subunit. Science 1990; 250: 121-3.

24 Payette P, Gossard F, Whiteway M, Dennis M. Expression and pharmacological characterization of the human M1 muscarinic receptor in Saccharomyces cerevisiae. FEBS Lett 1990; 266: 21-5.

25 Talmont F, Sidobre S, Demange P, Milon A, Emorine LJ. Expression and pharmacological characterization of the human mu-opioid receptor in the methylotrophic yeast Pichia pastoris. FEBS Lett 1996; 394: 268-72.

26 Beukers MW, Klaassen CH, De Grip WJ, Verzijl D, Timmerman H, Leurs R. Heterologous expression of rat epitope-tagged histamine $\mathrm{H} 2$ receptors in insect Sf9 cells. Br J Pharmacol 1997; 122: 867-74.

27 Weiss HM, Haase W, Reilander H. Expression of an integral membrane protein, the $5 \mathrm{HT}_{5 \mathrm{~A}}$ receptor. Methods Mol Biol 1998; 103: 227-39.

28 Sarramegna V, Talmont F, Seree de Roch M, Milon A, Demange P. Green fluorescent protein as a reporter of human mu-opioid receptor overexpression and localization in the methylotrophic yeast Pichia pastoris. J Biotechnol 2002; 99: 23-39.

29 Mollaaghababa R, Davidson FF, Kaiser C, Khorana HG. Structure and function in rhodopsin: expression of functional mammalian opsin in Saccharomyces cerevisiae. Proc Natl Acad Sci U S A 1996; 93: 11482-6.

30 Asada H, Uemura T, Yurugi-Kobayashi T, Shiroishi M, Shimamura T, Tsujimoto $\mathrm{H}$, et al. Evaluation of the pichia pastoris expression system for the production of GPCRs for structural analysis. Microb Cell Fact 2011; 10: 24.

31 O'Malley MA, Mancini JD, Young CL, McCusker EC, Raden D, Robinson AS. Progress toward heterologous expression of active G-proteincoupled receptors in Saccharomyces cerevisiae: Linking cellular stress response with translocation and trafficking. Protein Sci 2009; 18: $2356-70$.

32 Shukla AK, Haase W, Reinhart C, Michel H. Heterologous expression and comparative characterization of the human neuromedin $U$ subtype II receptor using the methylotrophic yeast Pichia pastoris and mammalian cells. Int J Biochem Cell Biol 2007; 39: 931-42.

33 Butz JA, Niebauer RT, Robinson AS. Co-expression of molecular chaperones does not improve the heterologous expression of mammalian G-protein coupled receptor expression in yeast. Biotechnol Bioeng 2003; 84: 292-304.

34 Zhang R, Kim TK, Qiao ZH, Cai J, Pierce WM Jr, Song ZH. Biochemical and mass spectrometric characterization of the human $\mathrm{CB} 2$ cannabinoid receptor expressed in Pichia pastoris - importance of correct processing of the N-terminus. Protein Expr Purif 2007; 55: 225-35.

35 Bertin B, Freissmuth M, Breyer RM, Schutz W, Strosberg AD, Marullo S. Functional expression of the human serotonin $5-\mathrm{HT}_{1 \mathrm{~A}}$ receptor in Escherichia coli. Ligand binding properties and interaction with recombinant G protein alpha-subunits. J Biol Chem 1992; 267: 8200-6.

36 Grisshammer R, Duckworth R, Henderson R. Expression of a rat neurotensin receptor in Escherichia coli. Biochem J 1993; 295: 5716.

37 Serrano-Vega MJ, Magnani F, Shibata Y, Tate CG. Conformational thermostabilization of the beta1-adrenergic receptor in a detergentresistant form. Proc Natl Acad Sci U S A 2008; 105: 877-82.

38 Sarkar CA, Dodevski I, Kenig M, Dudli S, Mohr A, Hermans E, et al. Directed evolution of a $\mathrm{G}$ protein-coupled receptor for expression, stability, and binding selectivity. Proc Natl Acad Sci U S A 2008; 105: 14808-13.

39 Dodevski I, Pluckthun A. Evolution of three human GPCRs for higher expression and stability. J Mol Biol 2011; 408: 599-615.

40 Rosenbaum DM, Cherezov V, Hanson MA, Rasmussen SG, Thian FS, Kobilka TS, et al. GPCR engineering yields high-resolution structural insights into beta2-adrenergic receptor function. Science 2007; 318: 1266-73.

41 Alexandrov Al, Mileni M, Chien EY, Hanson MA, Stevens RC. Microscale fluorescent thermal stability assay for membrane proteins. Structure 2008; 16: 351-9.

42 Rasmussen SG, Choi HJ, Rosenbaum DM, Kobilka TS, Thian FS, Edwards PC, et al. Crystal structure of the human beta2 adrenergic G-protein-coupled receptor. Nature 2007; 450: 383-7.

43 Ballesteros J, Weinstein $\mathrm{H}$. Integrated methods for the construction of three-dimensional models and computational probing of structurefunction relations in $\mathrm{G}$ protein-coupled receptors. Methods Neurosci 1995; 25: 366-428.

44 Roth CB, Hanson MA, Stevens RC. Stabilization of the human beta2adrenergic receptor TM4-TM3-TM5 helix interface by mutagenesis of Glu122(3.41), a critical residue in GPCR structure. J Mol Biol 2008; 376: 1305-19.

45 Lebon G, Bennett K, Jazayeri A, Tate CG. Thermostabilisation of an agonist-bound conformation of the human adenosine $A_{2 A}$ receptor. J Mol Biol 2011; 409: 298-310.

46 Shibata Y, White JF, Serrano-Vega MJ, Magnani F, Aloia AL, Grisshammer R, et al. Thermostabilization of the neurotensin receptor NTS1. J Mol Biol 2009; 390: 262-77.

47 Hong WX, Baker KA, Ma X, Stevens RC, Yeager M, Zhang Q. Design, synthesis, and properties of branch-chained maltoside detergents for stabilization and crystallization of integral membrane proteins: human connexin 26. Langmuir 2010; 26: 8690-6.

48 Chae PS, Rasmussen SG, Rana RR, Gotfryd K, Chandra R, Goren MA, et al. Maltose-neopentyl glycol (MNG) amphiphiles for solubilization, stabilization and crystallization of membrane proteins. Nat Methods 2010; 7: 1003-8.

49 Bayburt TH, Sligar SG. Self-assembly of single integral membrane proteins into soluble nanoscale phospholipid bilayers. Protein Sci 2003; 12: 2476-81.

50 Nath A, Atkins WM, Sligar SG. Applications of phospholipid bilayer nanodiscs in the study of membranes and membrane proteins. Biochemistry 2007; 46: 2059-69.

51 Tribet C, Audebert R, Popot JL. Amphipols: polymers that keep membrane proteins soluble in aqueous solutions. Proc Natl Acad Sci U S A 1996; 93: 15047-50.

52 Gorzelle BM, Hoffman AK, Keyes MH, Gray DN, Ray DG, Sanders CR. Amphipols can support the activity of a membrane enzyme. J Am Chem Soc 2002; 124: 11594-5.

53 Breyton C, Pucci B, Popot JL. Amphipols and fluorinated surfactants: Two alternatives to detergents for studying membrane proteins in vitro. Methods Mol Biol 2010; 601: 219-45. 
54 Schafmeister CE, Miercke LJ, Stroud RM. Structure at $2.5 \mathrm{~A}$ of a designed peptide that maintains solubility of membrane proteins. Science 1993; 262: 734-8.

55 McGregor CL, Chen L, Pomroy NC, Hwang P, Go S, Chakrabartty A, et al. Lipopeptide detergents designed for the structural study of membrane proteins. Nat Biotechnol 2003; 21: 171-6.

56 Kiley P, Zhao X, Vaughn M, Baldo MA, Bruce BD, Zhang S. Selfassembling peptide detergents stabilize isolated photosystem I on a dry surface for an extended time. PLoS Biol 2005; 3: e230.

57 Barthelemy P, Ameduri B, Chabaud E, Popot JL, Pucci B. Synthesis and preliminary assessments of ethyl-terminated perfluoroalkyl nonionic surfactants derived from tris(hydroxymethyl)acrylamidomethane. Org Lett 1999; 1: 1689-92.

58 McQuade DT, Quinn MA, Yu SM, Polans AS, Krebs MP, Gellman SH. Rigid amphiphiles for membrane protein manipulation. Angew Chem Int Ed Engl 2000; 39: 758-61.

59 Chae PS, Wander MJ, Bowling AP, Laible PD, Gellman SH. Glycotripod amphiphiles for solubilization and stabilization of a membrane-protein superassembly: importance of branching in the hydrophilic portion. Chembiochem 2008; 9: 1706-9.

60 Zhang Q, Ma X, Ward A, Hong WX, Jaakola VP, Stevens RC, et al. Designing facial amphiphiles for the stabilization of integral membrane proteins. Angew Chem Int Ed Engl 2007; 46: 7023-5.

61 Chae PS, Gotfryd K, Pacyna J, Miercke L, Rasmussen SG, Robbins RA, et al. Tandem facial amphiphiles for membrane protein stabilization. J Am Chem Soc 2010; 132: 16750-2.

62 Wu H, Su K, Guan X, Sublette ME, Stark RE. Assessing the size, stability, and utility of isotropically tumbling bicelle systems for structural biology. Biochim Biophys Acta 2010; 1798: 482-8.

63 Faham S, Bowie JU. Bicelle crystallization: a new method for crystallizing membrane proteins yields a monomeric bacteriorhodopsin structure. J Mol Biol 2002; 316: 1-6.

64 Arnold A, Labrot T, Oda R, Dufourc EJ. Cation modulation of bicelle size and magnetic alignment as revealed by solid-state NMR and electron microscopy. Biophys J 2002; 83: 2667-80.

65 Faham S, Boulting GL, Massey EA, Yohannan S, Yang D, Bowie JU. Crystallization of bacteriorhodopsin from bicelle formulations at room temperature. Protein Sci 2005; 14: 836-40.

66 Bayburt TH, Sligar SG. Membrane protein assembly into Nanodiscs. FEBS Lett 2010; 584: 1721-7.

67 Borch J, Hamann T. The nanodisc: a novel tool for membrane protein studies. Biol Chem 2009; 390: 805-14.

68 Leitz AJ, Bayburt TH, Barnakov AN, Springer BA, Sligar SG. Functional reconstitution of beta2-adrenergic receptors utilizing self-assembling Nanodisc technology. Biotechniques 2006; 40: 601-2.

69 Borch J, Roepstorff P, Moller-Jensen J. Nanodisc-based co-immunoprecipitation for mass spectrometric identification of membraneinteracting proteins. Mol Cell Proteomics 2011; 10: 0110.006775.

70 Wohri AB, Johansson LC, Wadsten-Hindrichsen P, Wahlgren WY, Fischer G, Horsefield R, et al. A lipidic-sponge phase screen for membrane protein crystallization. Structure 2008; 16: 1003-9.

71 Caffrey M. Crystallizing membrane proteins for structure determination: use of lipidic mesophases. Annu Rev Biophys 2009; 38: 29-51.

72 Caffrey M, Cherezov V. Crystallizing membrane proteins using lipidic mesophases. Nat Protoc 2009; 4: 706-31.

73 Li D, Lee J, Caffrey M. Crystallizing membrane proteins in lipidic mesophases. A host lipid screen. Cryst Growth Des 2011; 11: 530-7.
74 Qiu H, Caffrey M. Lyotropic and thermotropic phase behavior of hydrated monoacylglycerols: structure characterization of monovaccenin. J Phys Chem B 1998; 102: 4819-29.

75 Landau EM, Rosenbusch JP. Lipidic cubic phases: a novel concept for the crystallization of membrane proteins. Proc Natl Acad Sci U S A 1996; 93: 14532-5.

76 Pebay-Peyroula E, Rummel G, Rosenbusch JP, Landau EM. X-ray structure of bacteriorhodopsin at 2.5 angstroms from microcrystals grown in lipidic cubic phases. Science 1997; 277: 1676-81.

77 Cherezov V, Clogston J, Papiz MZ, Caffrey M. Room to move: crystallizing membrane proteins in swollen lipidic mesophases. J Mol Biol 2006; 357: 1605-18.

78 Grabe M, Neu J, Oster G, Nollert P. Protein interactions and membrane geometry. Biophys J 2003; 84: 854-68.

79 Templer RH, Seddon JM, Duesing PM. Modeling the phase behavior of the inverse hexagonal and inverse bicontinuous cubic phases in 2:1 fatty acid/phosphatidylcholine mixtures. J Phys Chem B 1998; 102: 7262-71.

80 Misquitta Y, Caffrey M. Detergents destabilize the cubic phase of monoolein: implications for membrane protein crystallization. Biophys J 2003; 85: 3084-96.

81 Wadsten-Hindrichsen P, Bender J, Unga J, Engstrom S. Aqueous self-assembly of phytantriol in ternary systems: effect of monoolein, distearoylphosphatidylglycerol and three water-miscible solvents. J Colloid Interface Sci 2007; 315: 701-13.

82 Cherezov V, Liu J, Griffith M, Hanson MA, Stevens RC. LCP-FRAP assay for pre-screening membrane proteins for in meso crystallization. Cryst Growth Des 2008; 8: 4307-15.

83 Xu F, Liu W, Hanson MA, Stevens RC, Cherezov V. Development of an automated high throughput LCP-FRAP assay to guide membrane protein crystallization in lipid mesophases. Cryst Growth Des 2011; 11: 1193-201.

84 Liu W, Hanson MA, Stevens RC, Cherezov V. LCP-Tm: an assay to measure and understand stability of membrane proteins in a membrane environment. Biophys J 2010; 98: 1539-48.

85 Cherezov V, Peddi A, Muthusubramaniam L, Zheng YF, Caffrey M. A robotic system for crystallizing membrane and soluble proteins in lipidic mesophases. Acta Crystallogr D Biol Crystallogr 2004; 60: 1795-807.

86 Bowler MW, Guijarro M, Petitdemange S, Baker I, Svensson O, Burghammer $\mathrm{M}$, et al. Diffraction cartography: applying microbeams to macromolecular crystallography sample evaluation and data collection. Acta Crystallogr D Biol Crystallogr 2010; 66: 855-64.

87 Kraft P, Bergamaschi A, Broennimann C, Dinapoli R, Eikenberry EF, Henrich B, et al. Performance of single-photon-counting PILATUS detector modules. J Synchrotron Radiat 2009; 16: 368-75.

88 Cherezov V, Hanson MA, Griffith MT, Hilgart MC, Sanishvili R, Nagarajan $\mathrm{V}$, et al. Rastering strategy for screening and centring of microcrystal samples of human membrane proteins with a sub10 microm size X-ray synchrotron beam. J R Soc Interface 2009; 6: S587-97.

89 Mader K, Marone F, Hintermuller C, Mikuljan G, Isenegger A, Stampanoni M. High-throughput full-automatic synchrotron-based tomographic microscopy. J Synchrotron Radiat 2011; 18: 117-24.

90 Joachimiak A. High-throughput crystallography for structural genomics. Curr Opin Struct Biol 2009; 19: 573-84. 